\section{Glutamatergic Hypothesis in Schizophrenia}

Vincenzo Rapisarda, Antonino Petralia, Concetta De Pasquale, Department of Psychiatry, University of Catania, Catania Italy

The Authors in this paper intend to try, as their experience, through a research, functional data about plaquettes carriage of glutamine in schizophrenic inpatients with Huber's Model of Basic Symptoms. The emersed data propose interesting considerations not only in the schizophrenic Pathology but also in other psychopathological spheres as Bipolar Disorders.
THE ANTISPASTIC TRIAZOLE MDL 27531 SELECTIVELY ENHANCED GABA-ACTIVATED CHLORIDE CURRENT IN SPINAL CORD MOTORNEURONS. C.J. Rogers, A.M. Ogden and J. M. Kane. Marion Merrell Dow Research Institute, Cincinnati, OH 45215.

The triazole MDL 27531, 4-methyl-3-methylsulfonyl-5phenyl-4H-1,2,4-triazole was identified as an antispastic agent selective for protection against strychnine-induced seizures and hyperreflexia. These compounds did not displace the binding of $\left[{ }^{3} \mathrm{H}\right]$ muscimol, $\left[{ }^{3} \mathrm{H}\right]$ flunitrazepam or $\left[{ }^{35} \mathrm{~S}\right] \mathrm{TBPS}$ to the $\mathrm{GABA} \mathrm{A}_{\mathrm{A}}$ receptor. The present experiments were performed to better understand the mechanism of action of this compound.

Whole cell and single channel patch clamp recordings were obtained from $\mathrm{GABA}_{\mathrm{A}}$-receptor activated chloride channels using cultured murine spinal cord motomeurons or rat hippocampal pyramidal neurons. GABA $(2-5 \mu \mathrm{M})$ or GABA plus MDL $27531(100 \mathrm{nM}$ or $1 \mu \mathrm{M})$ was applied from blunt micropipettes using positive pressure.

In spinal motomeurons, the application of GABA plus MDL 27531 (100nM) potentiated GABA-activated chloride currents relative to GABA alone. However, GABA plus MDL $27531(1 \mu \mathrm{M})$ did not change or slightly reduced GABA-activated chloride currents. No increase in chloride current was observed with GABA plus MDL 27531 on hippocampal pyramidal neurons.

These data indicate that MDL 27531 modulates GABAactivated chloride current selectively. This may be due to selective modulation of subunit configurations of the $G A B A_{A}$ receptor. The effect observed with higher concentrations of MDL 27531 may reflect either the modulation of different subunit configurations of the GABA receptor or interactive effects with additional binding sites. Preliminary studies indicate that MDL 27531 does not modulate glycine-activated chloride currents. Further studies are planned using cloned subunit configurations of the $G A B A_{A}$ receptor complex.

\section{COMBINED AGONIST-ANTAGONIST TREATMENT FOR NICOTINE AND OTHER DAUG DEPENDENCIES.}

Jed E. Rose. Ph.D. 1,2, Edward D. Levin, Ph.D.2, Frederique M. Behm 2, Eric C. Westman, M.D.1,3, Roy M. Stein, M.D. 1,2, James D. Lane, Ph.D.2, and Gail V. Ripka 2

${ }^{1} \mathrm{VA}$ Medical Center. Durham. NC 27705, ${ }^{2}$ Department of Psychiatry. Duke University. Durham. NC, ${ }^{3}$ Department of Medicine, Duke University, Dusham, NC

Co-administration of an agonist wüh an antagonist may regulate receptor activation, resulting in relief of withdrawal symptoms and blockade of drug reward. In one study, 12 smokers rated the rewarding effects of cigarette smoke after separale and combined administration of nicoline and the nicotinic antagonist mecamylamine. While each drug offset potential side elfects of the other, they acted in unison to aftenuate smoking reward. In a second study, 48 subjects participated in a randomized, double-blind, placebo-controlled smoking cessalion trial. Nicotine skin patch therapy (21 mg/day for 6-8 weeks) + oral mecamylamine (2.5-5 mg b.i.d. for 5 weeks) was compared to nicotine patch + placebo. Mecamylamine treatment began two weeks belore smoking cessation. Combined agonistantagonist treatment produced significantly higher continuous smoking abstinence than agonist-alone treatment: $50 \%$ vs $16.7 \%$ at seven weeks $(p=.015)$, $37.5 \%$ vs $12.5 \%$ at six months $(p=.046)$ and $37.5 \%$ vs $4.2 \%$ at twelve months $(p=.004)$. Concurrent agonistantagonist treatment may prove useful in treating other drug dependencies, and has potential advantages over irealmenl using agonists alone, antagonists alone or partial agonists. 